\title{
Comparison of Surgical Outcomes of Laparoscopic Adrenalectomy Between Patients With Nonfunctioning Adrenal Adenomas and Subclinical Cushing Syndrome: A Single-Center Experience
}

\author{
Minyong Kang, Minki Baek, Jonghoon Lee, Byong Chang Jeong
}

Department of Urology, Samsung Medical Center, Sungkyunkwan University School of Medicine, Seoul, Korea

Purpose: To examine and compare the perioperative outcomes of laparoscopic adrenalectomy (LA) and to determine its efficacy in patients with nonfunctioning adrenal adenomas (NFA) and subclinical Cushing syndrome (SCS).

Materials and Methods: We retrospectively analyzed the clinical data obtained from 79 consecutive patients who underwent LA for the treatment of either NFA $(n=35)$ or SCS $(n=44)$ between 2011 and 2016. All patients had undergone computed tomography, as well as endocrinological tests to confirm the diagnosis prior to the adrenalectomy. The primary endpoint was improved metabolic parameters relating to diabetes, hypertension, dyslipidemia, and obesity.

Results: Patients with SCS compared to those with NFA showed a higher occurrence of diabetes $(29.5 \%$ vs. $11.4 \%)$, hypertension (59.1\% vs. $34.3 \%)$, and dyslipidemia (43.2\% vs. $14.3 \%)$. Patients with SCS showed a smaller median tumor size compared to those with NFA $(2.5 \mathrm{~cm}$ vs. $5 \mathrm{~cm})$. No significant perioperative complications $\geq$ Clavien-Dindo classification grade III were observed in any patient (SCS or NFA group). In terms of their metabolic profile, patients with SCS showed a significant postoperative improvement in hypertension $(50.0 \%)$, diabetes $(53.9 \%)$, dyslipidemia (31.6\%), and obesity (29.2\%). However, patients with NFA showed a postoperative improvement only in dyslipidemia (40.0\%) and obesity $(4.8 \%)$.

Conclusions: Owing to absence of significant perioperative complications and the marked postoperative improvement in metabolic impairment, LA is a useful treatment strategy in patients diagnosed with SCS. In contrast, LA was not observed to show beneficial effects in correcting/improving the metabolic profile in patients presenting with NFA. (Korean J Urol Oncol 2017;15:137-142)

Key Words: Adrenal incidentaloma - Subclinical Cushing syndrome - Nonfunctioning adrenal adenoma Laparoscopic adrenalectomy $\cdot$ Surgical outcomes

Received October 7, 2017, Revised October 16, 2017,

Accepted October 22, 2017

Corresponding Author: Byong Chang Jeong

Department of Urology, Samsung Medical Center, Sungkyunkwan University School of Medicine, 81 Irwon-ro, Gangnam-gu, Seoul 06351, Korea

E-mail: bc2.Jung@samsung.com

Tel: +82-2-3410-3557, Fax: +82-2-3410-6992

ORCID code: https://orcid.org/0000-0002-5399-2184

\section{INTRODUCTION}

Most adrenal incidentalomas are nonfunctioning adrenal adenomas (NFA) without classical clinical signs and symptoms that are commonly observed with glucocorticoid hypersecretion. ${ }^{1}$ Subclinical Cushing syndrome (SCS) is also a common endocrine disease with an estimated incidence of approximately $20 \%$ in patients presenting with adrenal incidentalomas. ${ }^{2}$ The 3 criteria commonly used to diagnose SCS are: (1)

cc) (i) (\$) This is an Open Access article distributed under the terms of the Creative Commons Attribution Non-Commercial License (http://creativecommons.org/licenses/by-nc/4.0/) which permits unrestricted non-commercial use, distribution, and reproduction in any medium, provided the original work is properly cited. 2017 (C) Copyright The Korean Urological Oncology Society and The Korean Prostate Society. All Rights Reserved. 
incidental detection of an adrenal adenoma using an imaging modality, (2) no clear phenotypic findings associated with Cushing syndrome, (3) subtle biochemical abnormalities such as adrenocorticotropic hormone (ACTH)-independent secretion of cortisol. ${ }^{3}$ Patients with SCS do not demonstrate the typical clinical signs and symptoms of Cushing syndrome (CS), and a majority may demonstrate nonspecific findings including hypertension, dyslipidemia, osteoporosis, and glucose intolerance. ${ }^{4}$

Although undeniably, adrenalectomy is the treatment of choice for the management of CS, optimal management of NFA and SCS remains a controversial issue with a lack of consensus in terms of conservative and/or surgical treatment. ${ }^{2,5}$ Although a few patients presenting with NFA demonstrate mild elevation of cortisol secretion without any symptoms of overt CS, indicating a state of SCS, only a small percentage of patients with SCS progress toward the development of overt CS. ${ }^{6}$ Thus, the benefit of adrenalectomy for patients with NFA or SCS remains a controversial issue. A few authors have claimed that surgical therapy to treat these patients is associated with a higher risk of perioperative morbi-mortality than is observed with conservative therapy. However, a few others have reported that laparoscopic adrenalectomy (LA) is followed by normalization/improvement in the adverse metabolic profile noted in patients with NFA and SCS. ${ }^{4,7}$

A number of small-scale studies have been performed in patients with either NFA or SCS; however, few studies have compared the effects of a LA directly in patients with NFA and SCS. We examined the perioperative outcomes of LA to determine its efficacy in patients with NFA and SCS, focusing on a comparison of the postoperative metabolic improvement observed between these groups.

\section{MATERIALS AND METHODS}

\section{Study Population}

We retrospectively obtained and evaluated the clinical data of 79 consecutive patients who underwent LA for the treatment of either SCS $(n=44)$ or NFA $(n=35)$ and who were regularly followed up at the Samsung Medical Center between 2011 and 2016. Patients with either SCS or NFA underwent LA according to the following surgical indications: (1) abnormal hormone secretion regardless of size, (2) larger than $4 \mathrm{~cm}$ in size, (3) suspicion of malignancy in computed tomography scan or magnetic resonance imaging findings, (4) increase in size of the mass and suspicious findings of malignant tumor during the follow-up periods. All surgical procedures of LA were performed through transperitoneal approach based on a standard protocol followed at Samsung Medical Center. All clinical data were anonymized, and personal identifiers were eliminated. Written informed consent by the patients was waived due to a retrospective nature of our study. This study was approved by the Institutional Review Board of Samsung Medical Center (approval number: 2017-11-019-001).

\section{Study Design}

All patients underwent diagnostic computed tomography. Biochemical and endocrine evaluation of adrenal function was performed using the following laboratory tests for estimation of: serum cortisol, 24-hour urinary free cortisol, basal plasma $\mathrm{ACTH}, 24$-hour urinary excretion of vanillylmandelic acid, catecholamines and metanephrine, serum catecholamines, serum renin activity, and aldosterone. A dexamethasone suppression test was performed in patients showing elevated serum cortisol or 24-hour urinary free cortisol levels. All laboratory test results were normal in patients with NFA, whereas patients with SCS showed abnormal responses to at least 2 concomitant dexamethasone suppression tests in the absence of clinical signs of CS such as facial plethora, easy bruising, and proximal muscle weakness.

Clinical parameters evaluated were: age at the time of surgery, sex, body mass index (BMI) $\left(\mathrm{kg} / \mathrm{m}^{2}\right)$, the presence of metabolic abnormalities such as diabetes, hypertension, dyslipidemia and obesity, tumor size and laterality, operative time, estimated blood loss (EBL), type of laparoscopic surgery performed, perioperative complications, and length of postoperative hospitalization. Diabetes was defined as a fasting glucose level of $\geq 126 \mathrm{mg} / \mathrm{dL}$ and/or a 2-hour plasma glucose level of $\geq 200 \mathrm{mg} / \mathrm{dL}$ following a 75 -g oral glucose tolerance test or if patients reported a history of receiving antidiabetic drugs. Hypertension was defined as a systolic blood pressure of $\geq 140$ $\mathrm{mmHg}$ and/or diastolic blood pressure of $\geq 90 \mathrm{mmHg}$ or if patients reported a history of receiving antihypertensive treatment. Hyperlipidemia was defined as a total cholesterol level of $\geq$ $220 \mathrm{mg} / \mathrm{dL}$ or if patients reported a history of receiving cholesterol-lowering agents. Obesity was defined as a BMI of $\geq 25.0$ $\mathrm{kg} / \mathrm{m}^{2}$ based on the Clinical Practice Guidelines for Overweight and Obesity in Korea. ${ }^{9}$

All patients were followed-up at 1, 3, 6, and 12 months and 
then annually following the adrenalectomy, particularly for determining the improvement of diabetes mellitus, hypertension, dyslipidemia, and obesity. We defined the clinical improvements of metabolic abnormalities as follows: (1) When there was discontinuation of medications for diabetes, hypertension, or hyperlipidemia. (2) When there was a decrease in the number or doses of these medications. (3) When there was a postoperative weight loss for patients with obesity.

\section{Statistical Analysis}

The primary endpoint was an improvement in metabolic abnormalities. A descriptive analysis of patient characteristics was performed, and the data were presented as median values with interquartile range (IQR) or a percentage (\%) of events. The Student t-test (for continuous variables) and the chi-square test (for categorical variables) were used to determine the statistical significance between groups. A p-value of $<0.05$ was considered statistically significant. All statistical analyses were

Table 1. Baseline demographic data of patients with subclinical Cushing' syndrome and nonfunctioning adrenal adenoma

\begin{tabular}{lccr}
\hline \multicolumn{1}{c}{ Variable } & SCS (n=44) & NFA (n=35) & p-value \\
\hline Age (yr) & 53 & 49 & 0.531 \\
& $(46-60)$ & $(41-59)$ & 0.151 \\
Sex & & & \\
$\quad$ Men & $18(40.9)$ & $20(57.1)$ & \\
$\quad$ Women & $26(59.1)$ & $15(42.9)$ & 0.505 \\
Body mass index (kg/m $\left.{ }^{2}\right)$ & 25.5 & 26.5 & \\
& $(23.5-27.3)$ & $(23.3-28.8)$ & \\
Tumor laterality & & & 0.376 \\
Left & $27(61.4)$ & $18(51.4)$ & \\
Right & $17(38.6)$ & $17(48.6)$ & \\
Tumor size (cm) & 2.5 & 5 & $<0.001$ \\
& $(2.1-3.5)$ & $(3.5-7.0)$ & \\
Operative procedure & \multicolumn{3}{c}{0.281} \\
LESS-A & $7(15.9)$ & $9(25.7)$ & \\
CLA & $37(84.1)$ & $26(74.3)$ & \\
Conversion to open & $0(0)$ & $0(0)$ & \\
Operative time (min) & 90 & 110 & 0.001 \\
& $(75-102)$ & $(97.5-132.5)$ & \\
Estimated blood loss (mL) & 50 & 100 & 0.488 \\
Postoperative stay (day) & $(50-100)$ & $(50-150)$ & \\
Follow-up (mo) & $12(6-7)$ & $4(3-4.5)$ & $<0.001$ \\
\hline
\end{tabular}

Values are presented as median (interquartile range) or number (\%). SCS: subclinical Cushing' syndrome, NFA: nonfunctioning adrenal tumor, LESS-A: laparoendoscopic single-site adrenalectomy, CLA: conventional laparoscopic adrenalectomy. performed using IBM SPSS Statistics ver. 22.0 (IBM Co., Armonk, NY, USA).

\section{RESULTS}

Table 1 shows the baseline demographic data and perioperative outcomes. Additionally, the pathologic results of NFA are summarized in Supplementary Table 1. Patients with SCS showed a smaller median tumor size than those with NFA (2.5 cm [IQR, 2.1-3.5] vs. $5 \mathrm{~cm}$ [IQR, 3.5-7.0], respectively, p $<0.001)$. The median operative time was shorter in patients with SCS than in those with NFA (90 minutes [IQR, 75-102] vs. 110 minutes [IQR, 97.5-132.5], respectively, $\mathrm{p}=0.002$ ). Conversely, median duration of postoperative hospitalization was longer in patients with SCS compared to those with NFA (6 days [IQR, 5-7] vs. 4 days [IQR, 3-4.5]). However, there were no statistically significant differences observed with respect to other variables including age at the time of surgery, sex, tumor laterality and EBL between patients with SCS and NFA. Notably, patients with SCS compared to those with NFA demonstrated a higher occurrence of diabetes $(29.5 \%$ vs. $11.4 \%$, respectively), hypertension $(59.1 \%$ vs. $34.3 \%$, respectively), and dyslipidemia ( $43.2 \%$ vs. $14.3 \%$, respectively) (Table 2). No statistically significant difference was observed in the occurrence of overweight (BMI $\geq 25 \mathrm{~kg} / \mathrm{m}^{2}$ ) between the groups (Table 2).

Notably, there were no significant perioperative complications categorized as Clavien-Dindo classification grade III or higher in both groups of patients (those with SCS or NFA). Among 9 patients who demonstrated intraoperative complications, 4 demonstrated bleeding and 5 demonstrated an injury to the adjacent organs. Among those who demonstrated an in-

Table 2. Preoperative metabolic profiles of patients with subclinical Cushing' syndrome and nonfunctioning adrenal adenoma

\begin{tabular}{lccc}
\hline Metabolic impairment & SCS $(\mathrm{n}=44)$ & NFA $(\mathrm{n}=35)$ & $\mathrm{p}$-value \\
\hline Diabetes mellitus & $13(29.5)$ & $4(11.4)$ & 0.052 \\
Hypertension & $26(59.1)$ & $12(34.3)$ & 0.028 \\
Dyslipidemia & $19(43.2)$ & $5(14.3)$ & 0.006 \\
BMI $\geq 25\left(\mathrm{~kg} / \mathrm{m}^{2}\right)$ & $24(54.5)$ & $21(60.0)$ & 0.627 \\
\hline
\end{tabular}

Values are presented as number (\%).

SCS: subclinical Cushing' syndrome, NFA: nonfunctioning adrenal tumor, BMI: body mass index. 
Table 3. Comparison of postoperative metabolic improvement between patients with subclinical Cushing' syndrome and nonfunctioning adrenal adenoma

\begin{tabular}{lrcr}
\hline \multicolumn{1}{c}{ Variable } & SCS $(\mathrm{n}=44)$ & NFA $(\mathrm{n}=35)$ & p-value \\
\hline Diabetes mellitus & $7 / 13(53.9)$ & $0 / 4(0)$ & 0.016 \\
Hypertension & $13 / 26(50.0)$ & $0 / 12(0)$ & $<0.001$ \\
Dyslipidemia & $6 / 19(31.6)$ & $2 / 5(40.0)$ & 0.290 \\
BMI $\geq 25\left(\mathrm{~kg} / \mathrm{m}^{2}\right)$ & $7 / 24(29.2)$ & $1 / 21(4.8)$ & 0.070 \\
\hline
\end{tabular}

Values are presented as number of improved patients/number of patients with disease (\%).

SCS: subclinical Cushing' syndrome, NFA: nonfunctioning adrenal tumor, BMI: body mass index.

jury to the adjacent organs such as the liver and/or spleen, 2 patients belonged to the SCS and 3 to the NFA group. Bleeding was successfully controlled with the use of electrocoagulation and absorbable hemostatic materials. Additionally, 2 postoperative complications were observed: 1 paralytic ileus and 1 acute kidney injury. These complications were successfully controlled by conservative management without an invasive intervention.

We assessed the postoperative metabolic profile (Table 3 ) in all patients to determine whether various metabolic abnormalities had been normalized/improved after performing the adrenalectomy. Patients with SCS showed significant postoperative improvement in hypertension (50.0\% [13 of 26]), diabetes (53.9\% [7 of 13]), dyslipidemia (31.6\% [6 of 19]), and obesity (29.2\% [7 of 24]). In contrast, patients with NFA showed a significant postoperative improvement only in dyslipidemia (40.0\% [2 of 5]), and while only 1 patient with obesity (4.8\%) showed improvement, no patient showed normalization/improvement in hypertension and diabetes in this group.

\section{DISCUSSION}

Given the variability in diagnostic criteria and optimal management of SCS, the most appropriate treatment for SCS patients remains a highly controversial issue, ${ }^{10}$ although a few studies provide evidence that surgical treatment may be associated with a positive effect on the long-term clinical and metabolic outcomes in patients with SCS. ${ }^{11-15}$ However, the limitations of most studies cannot be ignored including multiple/diverse definitions of SCS, a high degree of variability in the definition of study end-points and surgical outcomes, and uncontrolled study designs. ${ }^{2}$ In this study, we reported that LA was an effective and safe treatment modality for the management of patients with SCS, particularly because it was not associated with any significant perioperative complications and patients demonstrated significant postoperative improvement in hypertension, diabetes, dyslipidemia, and overweight. However, LA was not associated with beneficial effects in patients with NFA. To our knowledge, ours is the first study assessing the outcomes of LA in Korean patients diagnosed with NFA and SCS, particularly with respect to a comparison of the outcomes of postoperative improvement in metabolic profile in these patients.

Similar to our findings, the first randomized controlled trial performed to study this subject reported that diabetes improved in $62.5 \%$ (5 of 8 ), hypertension in 67\% (12 of 18), dyslipidemia in $37.5 \%$ ( 3 of 8 ), and obesity in $50 \%$ ( 3 of 6 ) in the surgical group, whereas some patients demonstrated worsening of diabetes and hypertension in the conservative treatment group. ${ }^{11}$ Miyazato et al. ${ }^{12}$ have shown that LA used for the management of patients with SCS demonstrated significant clinical efficacy in improving hypertension (66.7\%), diabetes (47.1\%), and dyslipidemia (20.0\%). Iacobone et al. ${ }^{8}$ have demonstrated efficacy of LA in inducing remission of hormonal abnormalities and improvement of blood pressure, glycemic control, body weight, and health-related quality of life in patients with SCS. Chiodini et al. ${ }^{16}$ have reported that adrenalectomy was more beneficial for patients with SCS compared to conservative management, particularly in those with nonsecreting adenomas measuring $>$ $4 \mathrm{~cm}$ in diameter or those with a $>1-\mathrm{cm}$ increase in size of the mass noted during surveillance.

In contrast to these findings, the Semmelweis University group analyzed 125 patients with NFA, and clearly noted that surgical treatment failed to normalize or improve the abnormal metabolic profile observed in these patients. ${ }^{5}$ They also indicated that adrenalectomy did not reduce the rates of atherosclerosis-related morbi-mortality in patients with $\mathrm{NFA}^{5}$ Furthermore, an adrenalectomy was observed to be associated with a risk of postoperative adrenal insufficiency of the adrenal remnant, requiring cortisol replacement therapy. ${ }^{17} \mathrm{~A}$ recent systematic review of 28 selected papers described that postoperative adrenal insufficiency was observed in $65.3 \%$ among 248 patients with SCS. ${ }^{18}$

Interestingly, no mortality was observed and only minor perioperative complications were observed in patients with either SCS or NFA in our study. These results are in agreement with 
those of previous studies. Alesina et al. ${ }^{14}$ have reported a study showing a 0 mortality rate and no major intraoperative complications associated with an adrenalectomy. They reported only minor postoperative complications including segmental relaxation of the abdominal wall $(n=4)$ and hematoma formation $(n=1)$, which were successfully managed conservatively. ${ }^{14}$ Raffaelli et al. ${ }^{15}$ have also demonstrated that only 2 of 29 patients with SCS showed Clavien-Dindo classification grade II complications (1 patient with superficial phlebitis and 1 with postoperative pneumonia) following an adrenalectomy. To summarize, we reckon that LA is a feasible, safe and clinically effective method to treat patients with SCS and is associated with a low perioperative complication and 0 mortality rate.

Our study has some limitations: (1) Our study was a retrospective study; therefore, an unavoidable bias such as selection bias, missing clinical data, and potential misclassification of enrolled patients could have affected our results. (2) Our study included a small number of patients, and we used only a single tertiary center database. Further studies need to be planned using a multicenter database for conclusive validation of our findings. (3) The median follow-up duration was only 12 months (IQR, 6-27) and 5 months (IQR, 1-10) in patients with SCS and NFA, respectively. Therefore, long-term outcomes of LA could not be evaluated in these patients.

\section{CONCLUSIONS}

In summary, our results demonstrated that LA was an effective and safe option to treat patients with SCS, particularly because it was associated with no significant perioperative complications and significant postoperative improvement in metabolic abnormalities such as hypertension, diabetes, dyslipidemia and obesity. However, LA was not observed to show beneficial effects in correcting/improving the metabolic profile in patients presenting with NFA. Our study offers valuable information to determine an optimal therapeutic strategy for the management of patients with SCS or NFA who present with metabolic manifestations.

\section{CONFLICT OF INTEREST}

The authors claim no conflicts of interest.

\section{ACKNOWLEDGMENTS}

This work was supported by a research grant from the Korea Health Technology R\&D Project through the Korea Health Industry Development Institute (KHIDI), funded by the Ministry of Health \& Welfare, Republic of Korea (grant number: HI17C0025).

\section{SUPPLEMENTARY MATERIAL}

Supplementary Table 1 can be found via https://doi.org/ 10.22465/kjuo.2017.15.3.137

\section{REFERENCES}

1. Kastelan D, Kraljevic I, Dusek T, Knezevic N, Solak M, Gardijan B, et al. The clinical course of patients with adrenal incidentaloma: is it time to reconsider the current recommendations? Eur J Endocrinol 2015;173:275-82.

2. Terzolo M, Pia A, Reimondo G. Subclinical Cushing's syndrome: definition and management. Clin Endocrinol (Oxf) 2012;76:12-8.

3. Terzolo M, Bovio S, Reimondo G, Pia A, Osella G, Borretta G, et al. Subclinical Cushing's syndrome in adrenal incidentalomas. Endocrinol Metab Clin North Am 2005;34:423-39, x.

4. Mitchell IC, Auchus RJ, Juneja K, Chang AY, Holt SA, Snyder WH 3rd, et al. "Subclinical Cushing's syndrome" is not subclinical: improvement after adrenalectomy in 9 patients. Surgery 2007;142:900-5.

5. Sereg M, Szappanos A, Toke J, Karlinger K, Feldman K, Kaszper E, et al. Atherosclerotic risk factors and complications in patients with non-functioning adrenal adenomas treated with or without adrenalectomy: a long-term follow-up study. Eur J Endocrinol 2009;160:647-55.

6. Starker LF, Kunstman JW, Carling T. Subclinical Cushing syndrome: a review. Surg Clin North Am 2014;94:657-68.

7. Izaki H, Fukumori $T$, Takahashi $M$, Taue R, Kishimoto $T$, Tanimoto S, et al. Indications for laparoscopic adrenalectomy for non-functional adrenal tumor with hypertension: usefulness of adrenocortical scintigraphy. Int J Urol 2006;13: $677-81$.

8. Iacobone M, Citton M, Viel G, Boetto R, Bonadio I, Mondi I, et al. Adrenalectomy may improve cardiovascular and metabolic impairment and ameliorate quality of life in patients with adrenal incidentalomas and subclinical Cushing's syndrome. Surgery 2012;152:991-7.

9. Kim MK, Lee WY, Kang JH, Kang JH, Kim BT, Kim SM, et al. 2014 clinical practice guidelines for overweight and obe- 
sity in Korea. Endocrinol Metab (Seoul) 2014;29:405-9.

10. Zografos GN, Perysinakis I, Vassilatou E. Subclinical Cushing's syndrome: current concepts and trends. Hormones (Athens) 2014;13:323-37.

11. Toniato A, Merante-Boschin I, Opocher G, Pelizzo MR, Schiavi F, Ballotta E. Surgical versus conservative management for subclinical Cushing syndrome in adrenal incidentalomas: a prospective randomized study. Ann Surg 2009;249:388-91.

12. Miyazato M, Ishidoya S, Satoh F, Morimoto R, Kaiho Y, Yamada S, et al. Surgical outcomes of laparoscopic adrenalectomy for patients with Cushing's and subclinical Cushing's syndrome: a single center experience. Int Urol Nephrol 2011;43:975-81.

13. Agarwal S, Chand G, Agarwal A. Posterior retroperitoneoscopic adrenalectomy for clinical and subclinical Cushing's syndrome. World J Surg 2011;35:237.

14. Alesina PF, Hommeltenberg S, Meier B, Petersenn S, Lahner $\mathrm{H}$, Schmid KW, et al. Posterior retroperitoneoscopic adrena- lectomy for clinical and subclinical Cushing's syndrome. World J Surg 2010;34:1391-7.

15. Raffaelli M, De Crea C, D'Amato G, Gallucci P, Lombardi $\mathrm{CP}$, Bellantone R. Outcome of adrenalectomy for subclinical hypercortisolism and Cushing syndrome. Surgery 2017;161: 264-71.

16. Chiodini I, Morelli V, Salcuni AS, Eller-Vainicher C, Torlontano M, Coletti F, et al. Beneficial metabolic effects of prompt surgical treatment in patients with an adrenal incidentaloma causing biochemical hypercortisolism. J Clin Endocrinol Metab 2010;95:2736-45.

17. Chiodini I. Clinical review: diagnosis and treatment of subclinical hypercortisolism. J Clin Endocrinol Metab 2011;96: 1223-36.

18. Di Dalmazi G, Berr CM, Fassnacht M, Beuschlein F, Reincke M. Adrenal function after adrenalectomy for subclinical hypercortisolism and Cushing's syndrome: a systematic review of the literature. J Clin Endocrinol Metab 2014;99:2637-45. 